\title{
Um Modelo de Agente Pedagógico para o Treinamento Adaptativo da Habilidade Metacognitiva de Monitoramento do Conhecimento em Sistemas Tutores Inteligentes
}

\author{
Tiago Roberto Kautzmann, Patricia Jaques (orientadora)
}

Programa Interdisciplinar de Pós-Graduação em Computação Aplicada (PIPCA) Universidade do Vale do Rio dos Sinos (UNISINOS) - São Leopoldo - RS - Brasil

tkautzmann@gmail.com, pjaques@unisinos.br

\begin{abstract}
This work investigates the effects of training the metacognitive skill of knowledge monitoring in step-based intelligent tutoring systems, when this training is adapted to the learners' characteristics. An animated pedagogical agent that encourages students to reflect on their knowledge was developed and integrated into an algebraic intelligent tutoring system. Both content and frequency of agent interventions are adapted to the student's metacognitive skill level, their knowledge and their problem solving history. Other studies investigating the specific effects of training the knowledge monitoring skill when this training is adapted to student are unknown. The results of an experimental evaluation with control group and 63 students suggest that students who receive the agent training improve their knowledge monitoring skill and their performance in the domain.
\end{abstract}

Resumo. Esse trabalho investiga os efeitos do treinamento da habilidade metacognitiva de monitoramento do conhecimento em sistemas tutores inteligentes do tipo step-based, quando o treinamento é adaptado a caraterísticas do aluno. Um agente pedagógico animado que encoraja o aluno a refletir sobre seu conhecimento foi desenvolvido e integrado a um sistema tutor inteligente algébrico. Tanto o conteúdo quanto a frequência das intervenções do agente são adaptados ao nível metacognitivo de monitoramento do conhecimento do estudante, ao seu conhecimento no domínio e ao seu histórico de solução de problemas. Não se tem conhecimento de outros estudos que investigaram os efeitos específicos do treinamento da habilidade de monitoramento do conhecimento, quando este é adaptado ao aluno. Os resultados de uma avaliação experimental com grupo de controle com 63 alunos sugerem que os alunos que recebem o treinamento do agente melhoram a habilidade de monitoramento do conhecimento e o desempenho no domínio.

\section{Introdução}

A habilidade metacognitiva de monitoramento do conhecimento é a habilidade das pessoas de identificarem o que sabem e o que não sabem sobre determinado assunto. Esta habilidade é fundamental para a aquisição de outras habilidades metacognitivas e tem influência no sucesso da aprendizagem em todos os cenários acadêmicos [Tobias e Everson 2002]. Além disso, alunos que conseguem identificar o que sabem e o que não sabem sobre determinado domínio de conhecimento são mais propensos a: a) remediarem suas fraquezas de aprendizagem, mobilizando esforços para a aquisição dos 
conhecimentos deficitários [Fogarty 1994]; b) buscarem ajuda quando necessário [Stavrianopoulos 2007]; c) estudarem estrategicamente [Tobias e Everson 2002].

Diversos estudos investigaram como sistemas computacionais de aprendizagem podem melhorar as habilidades metacognitivas dos alunos. O modelo RA (Reflection Assistant Model) de Gama (2003, 2004) apresenta um modelo de treinamento da habilidade de monitoramento do conhecimento e de outras duas habilidades metacognitivas. $\mathrm{O}$ treinamento incita o aluno a refletir sobre seu conhecimento, porém todas as atividades ocorrem em uma sequência fixa, ou seja, não são adaptadas a características do aluno. Os resultados deste estudo foram inconclusivos. O trabalho de Pimentel, Omar e França (2005) apresentou os primeiros passos de uma proposta de agente que treina o monitoramento do conhecimento em sistemas tutores inteligentes (STIs), mas não foram encontradas publicações que indiquem a continuidade deste estudo. O trabalho de Aleven e colegas (2006) descreve o Help Tutor, um agente integrado a um sistema tutor que treina a habilidade de help-seeking, que é a habilidade de um indivíduo de buscar ajuda de professores, colegas, livros, entre outros, apenas quando esta ajuda é necessária. Este comportamento tem relação com o monitoramento do conhecimento, pois o comportamento adequado de help-seeking sugere que o aluno consiga diferenciar entre o que ele sabe e o que não sabe [Tobias e Everson 2002]. Demais trabalhos buscaram treinar outras habilidades metacognitivas, mas incitaram o aluno, de forma explícita, a refletir sobre seu conhecimento em dado momento da instrução [Puntambekar e Boulay 1997, Woolf et al 2002, Azevedo et al 2012]. Também foram considerados estudos que buscaram treinar outras habilidades metacognitivas e que não incitaram o aluno, de forma explícita, a refletir sobre seu conhecimento. Estes estudos também foram selecionados como trabalhos relacionados em função de treinarem habilidades metacognitivas sobrejacentes ao monitoramento do conhecimento e por utilizarem abordagens instrucionais similares aos utilizados no presente estudo, como prompts e feedbacks [Goodman et al 1997, Conati e VanLehn 2000, Ramandalahy, Vidal e Broisin 2010, Kramarski e Michallsky 2013, VanLehn et al 2014].

Apenas o trabalho de Gama (2003 e 2004) avaliou os efeitos do treinamento para uma melhora da habilidade de monitoramento do conhecimento, mas também treinou outras habilidades metacognitivas conjuntamente. Nenhum dos trabalhos relacionados avaliou exclusivamente a habilidade de monitoramento do conhecimento. Dessa forma, os efeitos isolados de uma instrução específica buscando encorajar os alunos a refletirem sobre o seu conhecimento para uma melhora da habilidade de monitoramento do conhecimento em sistemas computacionais de aprendizagem ainda são desconhecidos. Além disso, é conhecido que uma propriedade importante no treinamento metacognitivo em ambientes computacionais é a adaptação da instrução a características do aluno [Azevedo e Hadwin 2005]. Nenhum dos treinamentos dos trabalhos relacionados adapta ao aluno a etapa da instrução que incita o aprendiz a refletir sobre seu conhecimento.

O presente trabalho busca investigar os efeitos do treinamento adaptativo específico da habilidade metacognitiva do monitoramento do conhecimento em sistemas tutores inteligentes que acompanham os passos de solução do aluno (STIs step-based). O conteúdo e a frequência das intervenções do treinamento são adaptados às seguintes características do aluno: habilidade de monitoramento do conhecimento, desempenho no domínio e histórico de solução de problemas. O treinamento incita o aluno a refletir sobre seu conhecimento durante o processo de solução de problemas. Um modelo de agente pedagógico de treinamento da habilidade de monitoramento do conhecimento foi 
V Congresso Brasileiro de Informática na Educação (CBIE 2016)

Anais dos Workshops do V Congresso Brasileiro de Informática na Educação (CBIE 2016)

proposto. $\mathrm{O}$ modelo foi implementado como um agente pedagógico animado e integrado ao PAT2Math, um STI algébrico que acompanha os passos de solução de equações.

\section{Modelo de agente pedagógico de treinamento da habilidade metacognitiva de monitoramento do conhecimento}

O modelo de agente pedagógico proposto busca treinar a habilidade metacognitiva de monitoramento do conhecimento em STIs do tipo step-based através de ações que incitam os alunos a refletirem sobre seu conhecimento. As seguintes estratégias são adotadas: (1) incitar o aluno a identificar o que o problema está pedindo; (2) incitar o aluno a dedicar algum tempo para refletir sobre seu conhecimento antes de entrar com um novo passo de solução do problema; (3) incitar o aluno a refletir sobre problemas similares resolvidos anteriormente. Estas estratégias de reflexão são apresentadas e discutidas por Fogarty (1994), Verschaffel (1999), Polya (1957) e Gama (2004).

O treinamento adaptativo é uma importante característica do trabalho. Tanto o conteúdo quanto a frequência de intervenções do treinamento são adaptados às seguintes características do aluno: (1) nível da habilidade de monitoramento do conhecimento; (2) conhecimento no domínio; (3) histórico de solução de problemas. Neste contexto adaptativo, o agente proposto pode ser integrado a STIs que: (1) fornecem assistência passo-a-passo durante a solução dos problemas (STIs step-based); (2) mantém o registro do histórico de solução de problemas; (3) identificam o conhecimento que poderia ser aplicado pelo aluno no próximo passo; (4) identificam o conhecimento aplicado pelo aluno em cada passo do problema e; (5) inferem o conhecimento do aluno no domínio.

O treinamento do agente usa três tipos de ações que buscam incitar a reflexão dos alunos: (1) prompts, que são mensagens que incitam o aluno a refletir sobre seu conhecimento; (2) feedbacks, que são mensagens que notificam o aluno sobre algum comportamento inadequado e sobre seu nível corrente da habilidade de monitoramento do conhecimento; (3) self-explanations, que são atividades que incitam o aluno a escrever, com suas próprias palavras, sobre como ele monitorou seu conhecimento.

\subsection{Avaliação da habilidade de monitoramento do conhecimento}

Para fornecer o treinamento adaptativo, é necessário um mecanismo que infira o nível corrente da habilidade de monitoramento do conhecimento do aluno. O presente trabalho usou o instrumento Knowledge Monitoring Assessment (KMA), descrito por Tobias e Everson (2002 e 2009), que mede a habilidade do estudante de monitorar o próprio conhecimento. O KMA compara a avaliação do estudante sobre o próprio conhecimento para resolver um problema com o seu desempenho na solução do mesmo problema. $\mathrm{O}$ instrumento gera quatro escores relativos ao desempenho do aluno em monitorar o seu conhecimento: (a) o aluno avalia que possui conhecimento para resolver o próximo passo do problema e consegue resolver $(++)$; (b) o aluno avalia que possui conhecimento para resolver o próximo passo, mas não consegue resolver (+-); (c) o aluno avalia que não possui conhecimento para resolver o próximo passo, mas consegue resolver $(-+)$; e (d) o aluno avalia que não possui conhecimento para resolver o próximo passo e não consegue resolver (--). Os escores ++ (a) e -- (d) são relativos a avaliações precisas do aluno sobre seu conhecimento, enquanto que os escores +- (b) e -+ (c) são relativos a avaliações imprecisas. $\mathrm{O}$ agente usa estes escores para gerar um índice, chamado índice KMA, que mede a discrepância entre o conhecimento avaliado pelo aluno com o conhecimento demonstrado por ele. O índice KMA reflete o nível corrente da habilidade do aluno de 
V Congresso Brasileiro de Informática na Educação (CBIE 2016)

Anais dos Workshops do V Congresso Brasileiro de Informática na Educação (CBIE 2016)

monitorar o seu conhecimento. Este trabalho utiliza a fórmula do Coeficiente Hamman $(\mathrm{CH})$ para calcular o índice KMA, como sugerido por Tobias e Everson (2002):

$$
\mathrm{CH}=\frac{(\mathrm{a}+\mathrm{d})-(\mathrm{b}+\mathrm{c})}{(\mathrm{a}+\mathrm{d})+(\mathrm{b}+\mathrm{c})}
$$

A fórmula calcula a diferença da proporção de escores relativos a avaliações metacognitivas precisas e a proporção dos escores relativos a avaliações imprecisas. $\mathrm{O}$ índice KMA é um número real entre $-1 \mathrm{e}+1$, no qual +1 indica precisão no monitoramento do conhecimento, enquanto que -1 indica imprecisão. $O$ presente trabalho também classificou o índice KMA em duas categorias: satisfatório e insatisfatório, separados por um valor limiar $(+0,5)$. Valores iguais ou maiores ao limiar indicam índice KMA satisfatório. $\mathrm{O}$ valor limiar foi definido após a realização de testes piloto, objetivando fazer com que o índice KMA fosse classificado como satisfatório para valores altos o suficiente para expressar um bom e consistente desempenho metacognitivo.

\subsection{Mecanismos de funcionamento}

O agente tem dois mecanismos de funcionamento: (1) o ciclo interno, responsável pelo treinamento metacognitivo e (2) o ciclo externo, responsável por ativar o ciclo interno.

\subsubsection{Ciclo Externo}

Responsável por ativar o ciclo interno, o ciclo externo é sempre executado antes do aluno tentar resolver um novo passo do problema. A figura 1 apresenta o fluxo do ciclo externo. Duas estratégias são utilizadas pelo ciclo externo para decidir se o ciclo interno é ativado. A primeira estratégia é utilizada quando o índice KMA do aluno é insatisfatório. Um número pertencente ao intervalo de números reais do índice KMA, entre -1 e +1 , é aleatoriamente gerado usando uma função de probabilidade uniforme. Se o valor gerado for igual ou maior ao índice KMA corrente do aluno, o ciclo interno é ativado. Quanto maior o índice KMA, ou seja, quanto melhor a habilidade do aluno de monitorar seu conhecimento, menor será a probabilidade de ativação do ciclo interno. Assim, a frequência de ativação do ciclo interno é adaptada ao nível metacognitivo do aluno.

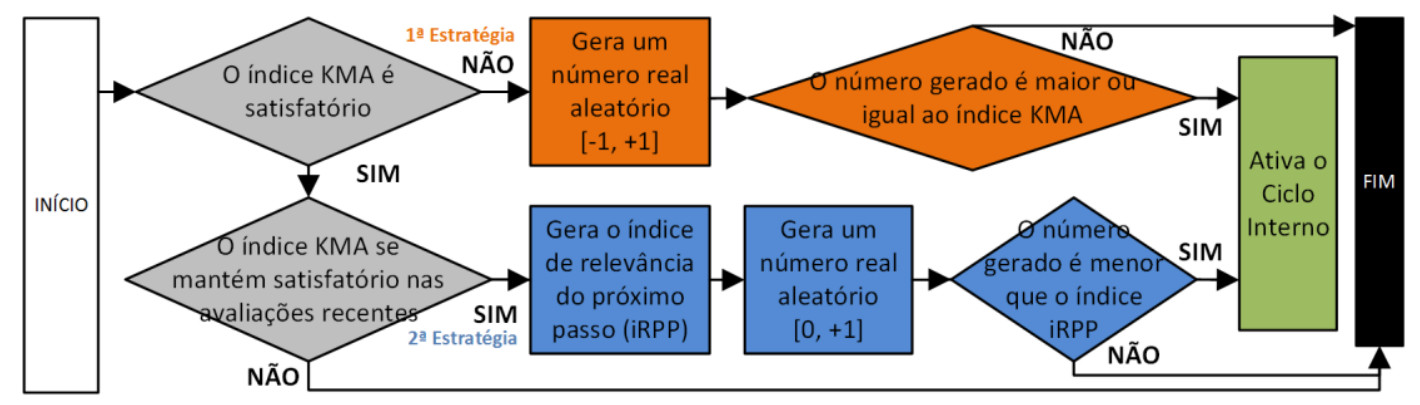

Figura 1. Fluxo do ciclo externo

A segunda estratégia do ciclo externo baseia-se no conhecimento do aprendiz no domínio, e é utilizada enquanto o aluno mantém o índice KMA satisfatório nas avaliações metacognitivas recentes. Evidências encontradas em testes piloto levaram o autor deste trabalho a acreditar que o aluno precisa refletir mais sobre seu conhecimento quando tenta resolver um problema que exige conhecimento que ele nem domina completamente e nem desconhece completamente. Esta evidência é resultante de observações visuais do autor em combinação com relatos de alunos durante os testes piloto. Assim, a segunda estratégia do ciclo externo utiliza a seguinte informação inferida pelo STI: probabilidade 
de conhecimento (valor real entre 0 e +1 ) do aluno em cada unidade de conhecimento possível de ser empregada no próximo passo do problema. Primeiramente, o mecanismo calcula um índice de relevância (iRUC) para cada unidade de conhecimento possível de ser aplicada pelo aluno no próximo passo. A fórmula do iRUC, proposta pelo presente trabalho, calcula a razão matemática da distância existente entre a probabilidade de conhecimento do aluno na unidade de conhecimento e o valor $0,5(50 \%)$ :

$$
\mathrm{iRUC}=1-\frac{\mid 0,5-\text { probConhecimento } \mid}{0,5}
$$

O mecanismo também gera o índice de relevância do próximo passo (iRPP), proposto pelo presente estudo, que é o valor médio dos iRUCs. O iRPP é um número real entre $0 \mathrm{e}+1$. Índices próximos de 0 indicam que o aluno provavelmente domina $\mathrm{o}$ conteúdo ou não tem conhecimento para o próximo passo. Por outro lado, índices de relevância próximos de +1 sugerem que o aluno possui uma probabilidade intermediária de conhecimento (próximo de 50\%) para resolver o próximo passo. Por fim, o mecanismo gera um valor aleatório entre $0 \mathrm{e}+1$, usando uma função de probabilidade uniforme. Caso o valor gerado for menor que o iRPP, o ciclo interno é ativado. Dessa forma, é mais provável que o ciclo interno seja ativado em passos que o aluno necessitaria refletir mais.

\subsubsection{Ciclo Interno}

O ciclo interno é responsável pelo treinamento da habilidade de monitoramento do conhecimento. A figura 2 apresenta o fluxo de ações e decisões do ciclo interno.

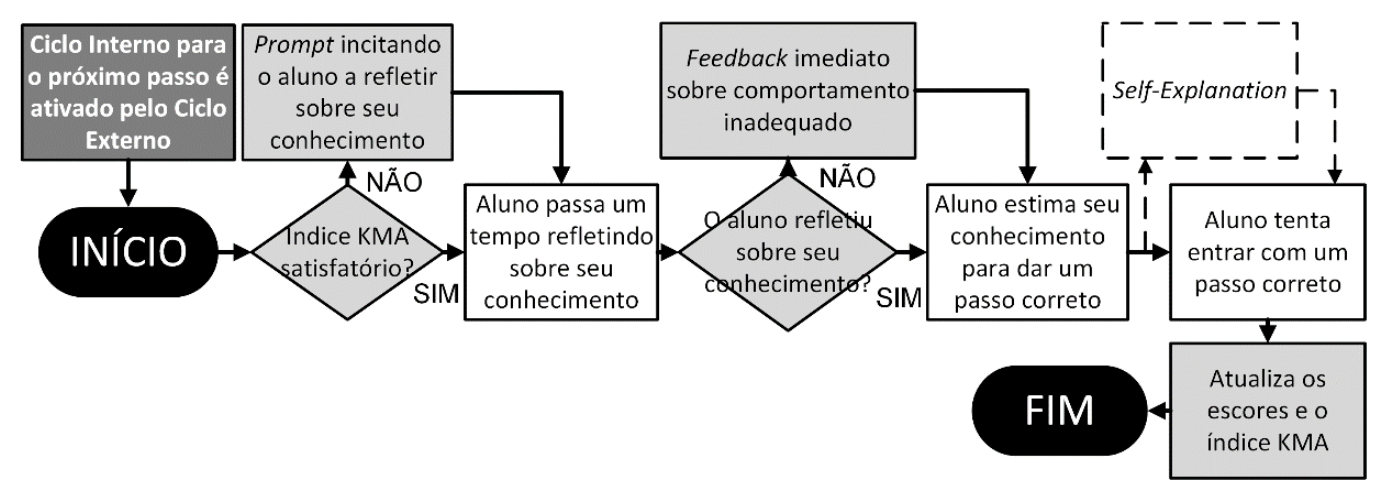

Figura 2. Fluxo do ciclo interno

Inicialmente, caso o índice KMA do aprendiz seja insatisfatório, o mecanismo seleciona um prompt que incita o aluno a refletir sobre seu conhecimento para o próximo passo do problema. O mecanismo aguarda pelo estudante. Se o aluno for muito reativo, ou seja, se após a apresentação do prompt o estudante tentar seguir para a próxima etapa do fluxo muito rapidamente, um feedback imediato é entregue ao aluno indicando seu comportamento inadequado. Na próxima etapa, o aluno deve estimar se tem conhecimento para resolver o passo, indicando "SIM" ou "NÃO". Na sequência, o aluno entra com sua solução para o passo do problema, permitindo que o mecanismo compare a estimativa de conhecimento do estudante para o próximo passo com o desempenho no mesmo passo, atualizando os escores KMA e o índice KMA. Além disso, o mecanismo pode, aleatoriamente, escolher entregar uma atividade de self-explanation em que o aluno preenche um formulário descrevendo como ele monitorou seu conhecimento.

Os prompts são mensagens de texto ativadas pelo ciclo interno que incitam o aluno a refletir sobre seu conhecimento. Os prompts foram agrupados em quatro níveis. $\mathrm{O}$ 
V Congresso Brasileiro de Informática na Educação (CBIE 2016)

Anais dos Workshops do V Congresso Brasileiro de Informática na Educação (CBIE 2016)

primeiro nível incita o estudante a refletir sobre seu conhecimento usando a descrição do problema corrente. O segundo nível incita o aluno a refletir sobre o conhecimento que ele já demonstrou dominar. O terceiro nível incita o aluno a refletir sobre passos que ele já resolveu e que utilizou conhecimento que poderá ser aplicado no próximo passo. O quarto nível apresenta ao aluno, na interface do sistema, um passo que ele resolveu anteriormente e que utilizou conhecimento que poderá ser empregado no próximo passo. Quanto menor o índice KMA, maior é o nível de prompt selecionado (mais próximo do quarto nível).

Quando o ciclo interno não é ativado e o índice KMA é insatisfatório, o agente poderá entregar (escolha aleatória) um feedback informando o nível corrente da habilidade do aluno de monitorar o seu conhecimento, apresentando dicas sobre como melhorar a habilidade metacognitiva de monitoramento do conhecimento.

\section{Avaliação}

Para fins de avaliação, o modelo de agente pedagógico foi implementado como um agente pedagógico animado (APA) representado por um personagem 2D feminino. O APA foi integrado ao PAT2Math (http://pat2math.unisinos.br), um STI que fornece assistência passo-a-passo (step-based) durante o processo de solução de equações de $1^{\circ}$ grau com uma incógnita. A figura 3 (a) mostra o agente, integrado ao PAT2Math, apresentando um prompt de nível quatro e (b) solicitando ao aluno que avalie seu conhecimento.
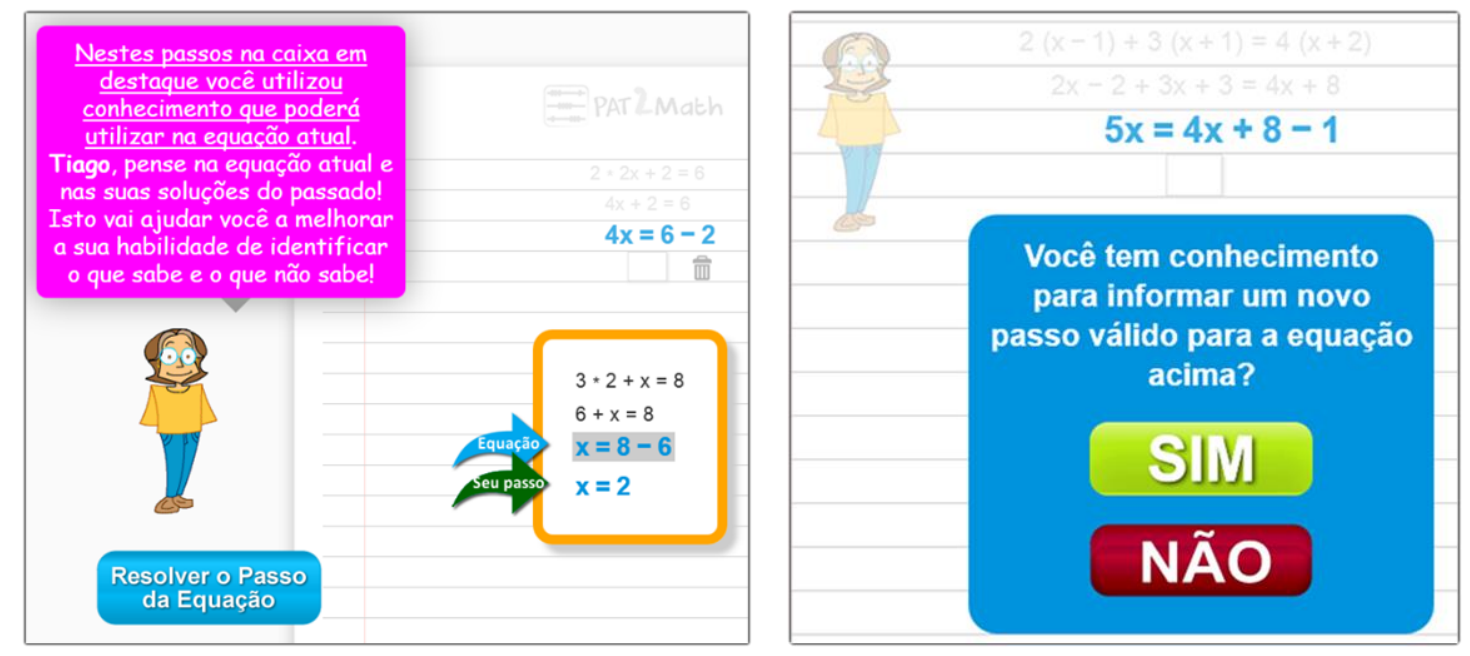

Figura 3. O agente (a) entrega um prompt (nível 4) que mostra um pas so similar resolvido anteriormente e (b) solicita ao aluno que estime seu conhecimento

O objetivo da avaliação é verificar se o treinamento do agente proposto melhora a habilidade do aluno de monitorar seu conhecimento. Mais especificamente, a avaliação objetiva responder as seguintes questões: (1) $O$ treinamento do agente melhora a habilidade de monitoramento do conhecimento do aluno?; (2) O treinamento do agente melhora a aprendizagem (desempenho no domínio) do aluno?; (3) é possível encontrar uma correlação entre o nível da habilidade de monitoramento do conhecimento e o desempenho no domínio quando o aluno recebe o treinamento do agente?

A principal hipótese levantada pelo trabalho é de que o treinamento adaptativo do agente efetivamente melhora a habilidade de monitoramento do conhecimento, pois incita o aluno a refletir sobre seu conhecimento, fazendo-o agir menos reativamente, e explica a importância da habilidade metacognitiva em seus estudos. Além disso, tanto a frequência quanto o conteúdo das intervenções do agente são adaptados de acordo com a 
habilidade de monitoramento do conhecimento do aprendiz, seu desempenho no domínio e seu histórico de solução de problemas. Esta característica adaptativa é fundamental no treinamento metacognitivo em ambientes computacionais de aprendizagem [Azevedo e Hadwin 2005] e ainda não foi verificada no treinamento da habilidade de monitoramento do conhecimento em ambientes computacionais de aprendizagem. $\mathrm{O}$ presente trabalho também levanta a hipótese de que o treinamento metacognitivo do agente melhora a aprendizagem e que uma correlação positiva pode ser encontrada entre a aprendizagem e a habilidade de monitoramento do conhecimento. Esta correlação já foi encontrada em sala de aula [Tobias e Everson 2002], mas ainda não em ambientes computacionais.

\subsection{Métodos e participantes}

Uma avaliação experimental com grupo de controle foi realizada com três turmas de $8^{\circ}$ Ano e uma turma de $7^{\circ}$ Ano do Ensino Fundamental em quatro escolas privadas do Rio Grande do Sul. A avaliação foi composta por um total de 6 a 7 sessões. Na primeira sessão, os alunos receberam um treinamento sobre o STI PAT2Math sem o agente metacognitivo. Na segunda sessão, os alunos retornaram o termo de consentimento (receberam na sessão anterior) assinados pelos pais e realizaram um pré-teste. Nas sessões seguintes (quatro sessões com duas turmas e apenas três sessões com as outras duas turmas, devido a indisponibilidade de calendário destas turmas) os alunos resolveram equações usando duas versões do PAT2Math nos laboratórios de informática das escolas. $\mathrm{Na}$ condição experimental, o agente pedagógico animado entregou a instrução metacognitiva. Na condição de controle, o agente foi modificado para não entregar ações metacognitivas, fornecendo apenas dicas relacionadas ao domínio, também entregues pelo agente da condição experimental. Na última sessão, os alunos participaram do pósteste. Cada sessão teve duração de 50 minutos e intervalo de uma semana entre as sessões.

Um total de 107 alunos, com idade entre 12 e 14 anos, participaram da avaliação e foram aleatoriamente atribuídos a dois grupos: experimental e controle. No entanto, foi possível considerar os dados de apenas 63 alunos que retornaram o termo de consentimento e que participaram das sessões de pré-teste e pós-teste. O grupo de controle foi composto por 34 alunos ( $44 \%$ de meninos e $56 \%$ de meninas), enquanto que o grupo experimental foi composto por 29 alunos (55\% de meninos e $45 \%$ de meninas).

Nas sessões de pré-teste, cada aluno recebeu uma folha de papel contendo dez equações algébricas. Para cada equação, o aluno deveria avaliar se tinha conhecimento para resolver. Após retornar a folha ao professor, cada aluno recebeu uma segunda folha de papel contendo as mesmas dez equações, mas desta vez deveria tentar resolver as equações. O mesmo procedimento foi realizado nas sessões de pós-teste, com diferentes equações e mesmo nível de dificuldade do pré-teste. Para avaliar a aprendizagem, cada aluno recebeu uma nota de desempenho no domínio (número inteiro entre 0 e 10), tanto no pré-teste, quanto no pós-teste. A avaliação do aluno sobre seu conhecimento e o seu desempenho no domínio foram utilizados para definir o índice KMA (descrito na seção 2.1) de cada estudante, tanto no pré-teste, quanto no pós-teste.

\section{Resultados e discussões}

Testes $t$ de Student compararam os índices KMA e as notas de desempenho. Testes com o coeficiente de Pearson verificaram a correlação entre os índices KMA e as notas no domínio. A confiabilidade dos dados amostrais foi testada através de testes Ryan-joiner para a normalidade dos dados e testes de Levene para a homogeneidade das variâncias. 
Um teste $t$ de Student de amostras independentes $(\alpha=.05)$ comparando a média dos índices KMA dos alunos do grupo experimental $(\mu=.800, \sigma=.251)$ com a média do grupo de controle $(\mu=.605, \sigma=.413)$ no pós-teste encontrou uma média significativamente superior $(t(61)=2.214, p=.015)$ para o grupo experimental. Um segundo teste $t$ de amostras independentes comparou os ganhos do índice KMA (diferença dos índices entre o pós-teste e o pré-teste) dos grupos. Um resultado estatístico marginal ao nível de confiança foi encontrado $(t(61)=1.588, p=.059)$, indicando que os ganhos de índice KMA no grupo experimental $(\mu=.231, \sigma=.373)$ foram superiores aos ganhos do grupo de controle $(\mu=.061, \sigma=.463)$. Um terceiro teste $t$ de amostras pareadas comparou os índices KMA entre o pré-teste e o pós-teste dentro de cada grupo e não encontrou diferenças significativas $(t(33)=.766, p=.225)$ entre o pós-teste $(\mu=.605, \sigma$ $=.413)$ e o pré-teste $(\mu=.544, \sigma=.325)$ no grupo de controle. No entanto, uma média significativamente superior $(t(28)=3.333, p<.01)$ foi encontrada no pós-teste $(\mu=.800$, $\sigma=.251)$ quando comparada ao pré-teste $(\mu=.569, \sigma=.381)$, no grupo experimental.

Um teste $t$ de amostras independentes $(\alpha=.05)$ comparou as notas no domínio entre os grupos no pós-teste. Uma média superior $(t(61)=2.327, p=0.012)$ foi encontrada no grupo experimental $(\mu=8.621, \sigma=1.741)$ em relação ao grupo de controle $(\mu=7.441$, $\sigma=2.205$ ). Um segundo teste $t$ de amostras independentes comparou os ganhos das notas no domínio (diferença das notas entre o pós-teste e o pré-teste) entre os grupos. A média do grupo experimental $(\mu=2.414, \sigma=2.147)$ foi superior à do grupo de controle $(\mu=$ $1.941, \sigma=1.825)$, mas esta diferença não foi significativa $(t(61)=.945, p=.174)$.

Testes de correlação no grupo experimental encontraram uma forte correlação positiva entre o índice KMA e o desempenho no domínio, no pós-teste, e uma moderada correlação positiva entre os ganhos de índice KMA e os ganhos de desempenho. Os mesmos testes encontraram uma fraca correlação positiva no grupo de controle.

Os resultados dos testes estatísticos apresentaram evidências positivas que corroboram com a hipótese de que o treinamento fornecido pelo agente pode melhorar a habilidade dos alunos de monitorarem seu conhecimento. Todos os testes indicaram que os alunos da condição experimental obtiveram índices metacognitivos superiores aos alunos da condição de controle. Outros testes apresentaram evidências que apoiam a hipótese de que a instrução do agente pode melhorar o desempenho no domínio, embora os resultados desses testes não tenham sido tão significativos. Também foram encontradas evidências de uma forte correlação positiva entre a habilidade de monitoramento do conhecimento e o desempenho no domínio no grupo experimental, indicando um forte relacionamento quando estas variáveis são observadas juntas. Esta evidência corrobora com resultados encontrados em sala de aula [Tobias e Everson 2002]. No entanto, estes resultados ainda não haviam sido verificados em ambientes computacionais.

Sabe-se que um dos princípios fundamentais para o sucesso da instrução metacognitiva é que o treinamento das habilidades metacognitivas deve ser prolongado [Veenman et al 2006]. Dessa forma, os resultados poderiam ter sido ainda melhores se os alunos tivessem participado de mais sessões de treinamento (participaram de três a quatro sessões). A quantidade de sessões não foi maior devido a restrições de agenda das escolas.

\section{Conclusões}

Este trabalho investigou os efeitos de um treinamento adaptativo da habilidade metacognitiva de monitoramento do conhecimento em sistemas tutores inteligentes do 
tipo step-based. Para alcançar os objetivos do estudo, um modelo de agente pedagógico foi proposto. O diferencial do presente trabalho em relação aos trabalhos relacionados é a investigação dos efeitos isolados de um treinamento adaptativo que incita o aluno a refletir sobre seu conhecimento para promover a habilidade metacognitiva de monitoramento do conhecimento. Os trabalhos relacionados buscaram treinar outras habilidades metacognitivas conjuntamente. O presente estudo obteve os primeiros resultados de um treinamento específico da habilidade de monitoramento do conhecimento em STIs. Além disso, os trabalhos relacionados não treinaram a habilidade de monitoramento do conhecimento de forma que a etapa da instrução que incita o aluno a refletir sobre seu conhecimento fosse adaptada a características do aluno.

$\mathrm{O}$ agente proposto foi implementado como um agente pedagógico animado e integrado ao PAT2Math, um STI do tipo step-based que assiste os alunos na solução de equações de $1^{\circ}$ grau com uma incógnita. Os resultados da avaliação experimental sugerem que o treinamento adaptativo do agente melhora a habilidade metacognitiva de monitoramento do conhecimento e o desempenho dos alunos no domínio. Além disso, uma forte correlação positiva entre a habilidade metacognitiva de monitoramento do conhecimento e o desempenho no domínio foi encontrada quando os alunos receberam o treinamento. Esta correlação já havia sido encontrada em sala de aula, mas ainda não em ambientes computacionais de aprendizagem.

Para medir a habilidade metacognitiva de monitoramento do conhecimento, o instrumento KMA não considera um viés cognitivo conhecido como excesso de confiança, em que a confiança de uma pessoa em seus julgamentos excede a precisão destes julgamentos. O presente estudo também não considera os estados afetivos do aluno, como as emoções, o humor e o engajamento. Trabalhos futuros pretendem investigar como o excesso de confiança e os estados afetivos interferem na habilidade de monitoramento do conhecimento e no treinamento desta habilidade, permitindo a criação de novos mecanismos de medição da habilidade metacognitiva e o melhoramento do treinamento desta habilidade em ambientes computacionais de aprendizagem.

\section{Referências}

Aleven, V., Mclaren, B., Koedinger, K. e Rool, I. (2006) Toward Meta-Cognitive Tutoring: A Model of Help-Seeking with a Cognitive Tutor. International Journal of Artificial Intelligence in Education, vol. 16, p. 101-130.

Azevedo, R. e Hadwin, A. F. (2005) Scaffolding Self-regulated Learning and Metacognition. Instructional Science, v. 33, n. 5-6, p. 367-379.

Azevedo, R., Feyzi-Behnagh, R. F., Duffy, M., Harley, J. M. e Trevors, G. (2012) Metacognition and self-regulated learning in student-centered leaning environments. In: D. Jonassen e S. Land (Eds.) Theoretical foundations of student-center learning environments, New York: Routledge, n. 2, p. 171-197.

Conati, C. e Van Lehn, K. (2000) Toward computer-based support of meta-cognitive skills: A computational framework to coach self-explanation. International Journal of Artificial Intelligence in Education, Amsterdam, v. 11, n. 1, p. 389-415.

Fogarty, R. (1994) How to Teach for Metacognitive Reflection. Pearson, Glenview.

Gama, C. (2003) PAL Tool: uma ferramenta cognitiva para organização e representação de problemas algébricos. In Anais do SBIE 2003, Rio de Janeiro, RJ. SBC. 
V Congresso Brasileiro de Informática na Educação (CBIE 2016)

Anais dos Workshops do V Congresso Brasileiro de Informática na Educação (CBIE 2016)

Gama, C. A. (2004) Integrating Metacognition Instruction in Interactive Learning Environments. Thesis (PhD), University of Sussex, Brighton.

Goodman, B., Soller, A., Linton, F. e Gaimari, R. (1997) Encouraging student reflection and articulation using a learning companion. International Journal of Artificial Intelligence in Education, Amsterdam, p. 151-158.

Gürer, D. (1998) The use of distributed agents in intelligent tutoring. In: Proceedings of 2nd ITS Workshop on Pedagogical Agents, San Antonio, Texas, p. 20-25.

Jennings, N., Sycara, K. e Wooldridge, M. (1998) A Roadmap of agent research and development. Journal of Autonomous Agents and Multi-Agent Systems, p. 275-306.

Kramarski, B. e Michalsky, T. (2013) Student and Teacher Perspectives on IMPROVE Self-Regulation Prompts in Web-Based Learning. In: R. Azevedo e V. Aleven (Eds.) International Handbook of Metacognition and Learning, Springer, NY.

Pimentel, E. P., Omar, N. e França, V. F. de (2005) Um modelo para Incorporação de Automonitoramento da Aprendizagem em STI. Revista Brasileira de Informática na Educação, Porto Alegre, v. 13, n. 1, p. 62-70.

Polya, G. (1957) How to Solve It. Princeton University Press, Garden City, NY.

Puntambekar, S. e Boulay, B. D. (1997) Design and Development of MIST - A System to Help Students Develop Metacognition. J. Educational Computing Research, Atlanta, USA, v. 16, n. 1, p. 1-35.

Ramandalahy, T., Vidal, P. e Broisin, J. (2010) An intelligent tutoring system supporting metacognition and sharing learners' experiences. In: Aleven, V., Kay, J. e Mostow, J. (Eds.) Lecture Notes in Computer Science, p. 402-404.

Stavrianopoulous, K. (2007) Adolescent's Metacognitive Knowledge Monitoring and Academic Help Seeking. College Student Journal, v. 41, n. 2, p. 444-453.

Tobias, S. e Everson, H. (2002) Knowing What You Know and What You Don't: Further Research on Metacognitive Knowledge Monitoring. Research Report, The College Board, New York, n. 2002-3, p. 1-25.

Tobias, S. e Everson, H. (2009) The Importance of Knowing What You Know: A Knowledge Monitoring Framework for Studying Metacognition. In: D. J. Hacker, J. Dunlosky e A. C. Graesser (Eds.) Handbook of Metacognition in Education, NY.

VanLehn, K., Burleson, W., Girard, S., Chavez-Echeagaray, M. E., Gonzalez-Sanchez, J., Hidalgo-Pontet, Y. e Zhang, L. (2014) The Affective Meta-Tutoring Project: Lessons Learned. In: Proceedings of ITS 2014, New York, NY, p. 84-93.

Veenman, M. V. J., Hout-Wolters, B. H. A. M. Van e Afflerbach, P. (2006) Metacognition and learning: conceptual and methodological considerations. Metacognition Learning, v.1, n. 1, p. 3-14.

Verschaffel, L. (1999) Realistic mathematical modelling and problem solving in the upper elementary school: analysis and improvement. In: J. H. M. Hamers, J. E. H. Luit, B. Csapó (Eds.) Teaching and learning thinking skills, Lisse, The Netherlands.

Woolf, B. P., Reid, J., Stillings, N., Bruno, M., Murray, D., Reese, P., Peterfreund, A. e Rath, K. (2002) A General Platform for Inquiry Learning. In: International Conference of Intelligent Tutoring Systems, Springer Berlin Heidelberg, NY. 